\title{
Trajectories of Scores on a Screening Instrument for PTSD Among World Trade Center Rescue, Recovery, and Clean-Up Workers
}

\author{
Carey B. Maslow, ${ }^{1}$ Kimberly Caramanica, ${ }^{1}$ Alice E. Welch, ${ }^{1}$ Steven D. Stellman, ${ }^{1,2}$ Robert M. Brackbill, ${ }^{1}$ \\ and Mark R. Farfel ${ }^{1}$ \\ ${ }^{1}$ New York City Department of Health and Mental Hygiene, World Trade Center Health Registry, Long Island City, New York, \\ USA \\ ${ }^{2}$ Department of Epidemiology, Mailman School of Public Health, Columbia University, New York, New York, USA
}

\begin{abstract}
The longitudinal course of posttraumatic stress disorder (PTSD) over 8-9 years was examined among 16,488 rescue and recovery workers who responded to the events of September 11, 2001 (9/11) at the World Trade Center (WTC; New York, NY), and were enrolled in the World Trade Center Health Registry. Latent class growth analysis identified 5 groups of rescue and recovery workers with similar score trajectories at 3 administrations of the PTSD Checklist (PCL): low-stable (53.3\%), moderate- stable (28.7\%), moderate-increasing (6.4\%), high-decreasing $(7.7 \%)$, and high-stable (4.0\%). Relative to the low-stable group, membership in higher risk groups was associated with 9/11-related exposures including duration of WTC work, with adjusted odds ratios ranging from 1.3 to 2.0, witnessing of horrific events (range $=1.3$ to 2.1 ), being injured (range $=1.4$ to 2.3 ), perceiving threat to life or safety (range $=2.2$ to 5.2 ), bereavement (range $=1.6$ to 4.8 ), and job loss due to $9 / 11$ (range $=2.4$ to 15.8 ). Within groups, higher PCL scores were associated with adverse social circumstances including lower social support, with $B$ coefficients ranging from 0.2 to 0.6 , divorce, separation, or widowhood (range $=0.4-0.7$ ), and unemployment (range $=0.4-0.5$ ). Given baseline, exposure-related, and contextual influences that affect divergent PTSD trajectories, screening for both PTSD and adverse circumstances should occur immediately, and at regular intervals postdisaster.
\end{abstract}

Disasters of intentional origin, like the events of September 11, 2001 (9/11), may elicit emotional responses that are of greater severity (Galea, Nandi, \& Vlahov, 2005), are qualitatively different (Bills et al., 2008), and result in higher rates of posttraumatic stress disorder (PTSD) than do those of unintentional human or natural origin (Cukor et al., 2011). Rescue and recovery workers may be at higher risk of mental health sequelae than are other populations exposed to the same disaster (Bills et al., 2008). Among the estimated 90,000 rescue and recovery workers responding to the events of 9/11 (Murphy

This study was supported by Cooperative Agreement Numbers 5U50/OH009739 and 1E11/OH009630 from National Institute for Occupational Safety and Health-Centers for Disease Control (NIOSH-CDC); U50/ATU272750 from the Agency for Toxic Substances and Disease Registry(ATSDR-) CDC, which included support from the National Center for Environmental Health- (NCEH-) CDC; and the New York City Department of Health and Mental Hygiene (NYC DOHMH). Its contents are solely the responsibility of the authors and do not necessarily represent the official views of NIOSH-CDC.

Correspondence concerning this article should be addressed to Carey B. Maslow, World Trade Center Health Registry, New York City Department of Health and Mental Hygiene, 42-09 28th Street, Long Island City, NY 11101. E-mail: cmaslow@health.nyc.gov

(C) 2015 International Society for Traumatic Stress Studies. View this article online at wileyonlinelibrary.com

DOI: $10.1002 /$ jts.22011 et al., 2007), elevated rates of PTSD have been reported since shortly after the disaster, and associated with specific exposures incurred on 9/11 (Chiu et al., 2011; Corrigan et al., 2009; Gross et al., 2006; Levin et al., 2004). Over time, changes in individual characteristics associated with PTSD affect its development within individuals and divergence of developmental trajectories across individuals. Over a decade after the event, however, relatively few studies have examined patterns of development of PTSD among rescue and recovery workers, and fewer still (Bowler et al., 2012) have considered the effects on its course of contextual changes occurring coincidentally, or as a result of PTSD. To our knowledge, none has assessed the course of PTSD within multiple subsets of rescue and recovery workers defined by occupation, although prevalence may vary considerably (Perrin et al., 2007).

This study described the course of PTSD symptoms in a large, diverse cohort of rescue and recovery workers enrolled in the World Trade Center Health Registry (Registry) by examining scores on a screening instrument administered on three occasions over an 8-9-year period. It identified groups of workers defined by similar score trajectories, and sociodemographic, 9/11-related exposure, and other descriptors associated with membership in each trajectory group. It also assessed the impact of correlates that could have changed over time (time-dependent covariates) within trajectory groups. 


\section{Method}

\section{Participants and Procedure}

The Registry is a prospective cohort of individuals with high likelihood of exposure to the events of 9/11 (Farfel et al., 2008) who consented to participate after being informed of the Registry's purposes. Surveys administered 3-4 years apart elicited information about exposures, physical and mental health, health-care utilization, and measures of functional status. To date, three waves of data collection have occurred: in 2003-2004 (Wave 1), 2007-2008 (Wave 2), and 2011-2012 (Wave 3). Enrollees who were at least 18 years of age at Wave 1, performed rescue/recovery work at the World Trade Center (WTC) site between 9/11 and June 30, 2002, and completed all three surveys were included in analyses. The final sample comprised 16,488 rescue and recovery workers, and included firefighters (14.5\%), police (14.2\%), emergency medical services/medical/disaster personnel $(7.4 \%)$, construction/engineering workers (12.9\%), affiliated (17.8\%) and unaffiliated $(12.1 \%)$ volunteers, other government workers (15.8\%), and sanitation workers (5.3\%). The majority was non-Hispanic White $(77.5 \%)$, above 35 years of age at Wave $1(81.3 \%)$, and male $(78.8 \%$; Table 1). Most had graduated from high school (74.4\%), earned $\geq \$ 75,000$ in 2002 (52.9\%), were married or living with a partner (73.3\%), and reported medium or high levels of social support (78.3\%). A small proportion (7.7\%) reported a pre-9/11 diagnosis of PTSD, anxiety, or depression; nearly one third $(32.6 \%)$ reported having seen a professional and/or taken medication for a mental health problem within one year of Wave 2 and/or Wave 3. Nearly half (47.2\%) reported having experienced $\geq$ one of the following traumas prior to 9/11: a natural/human-made disaster; a serious accident; an attack with a weapon or without a weapon, but with intent to kill or seriously injure; unwanted sexual contact by force or threat of force; another situation in which one feared being, or was seriously injured or killed; or witnessing others in a situation in which they feared or were seriously injured or killed.

Most (66\%) reported no exposure to the dust cloud; one quarter $(24.5 \%)$ reported intense exposure. Nearly half $(45.5 \%)$ reported having worked on the pile of debris that resulted from collapsed buildings; under 3\% reported having been in a building on 9/11 before, during, and/or after it collapsed.

\section{Measures}

PTSD symptomatology was assessed at each wave by summing responses to the PTSD Checklist-Civilian Version (PCL; Weathers, Huska, \& Keane, 1991), adapted to be specific to the events of 9/11. The PCL is a self-report checklist on which each of 17 items is rated on a scale from $1=$ Not at all to $5=$ Extremely. Scale items correspond to symptom criteria defined in the Diagnostic and Statistical Manual of Mental Disorders (4th ed., text rev.; DSM-IV-TR; American Psychiatric Association, 2000). The PCL has demonstrated strong psychometric properties, with coefficients for internal consistency as high as
Table 1

Sample Description: Rescue/Recovery Workers in the World Trade Center Health Registry, Waves 1, 2, and 3

\begin{tabular}{|c|c|c|}
\hline Variable & $n$ & $\%$ \\
\hline \multicolumn{3}{|l|}{ Race/ethnicity } \\
\hline Non-Hispanic White & 12,781 & 77.5 \\
\hline Non-Hispanic Black & 1,047 & 6.4 \\
\hline Hispanic or Latino & 1,810 & 11.0 \\
\hline Asian/ multiracial/other & 850 & 5.2 \\
\hline \multicolumn{3}{|l|}{ Age at Wave 1 (years) } \\
\hline $18-35$ & 3,086 & 18.7 \\
\hline $36-45$ & 6,029 & 36.6 \\
\hline $46-55$ & 4,968 & 30.1 \\
\hline$\geq 56$ & 2,405 & 14.6 \\
\hline \multicolumn{3}{|l|}{ Gender } \\
\hline Male & 12,985 & 78.8 \\
\hline Female & 3,503 & 21.2 \\
\hline \multicolumn{3}{|l|}{ Educational attainment $^{\mathrm{a}}$} \\
\hline High-school graduate or less & 4,216 & 25.7 \\
\hline Some college & 5,177 & 31.6 \\
\hline College/postgraduate & 7,016 & 42.8 \\
\hline \multicolumn{3}{|l|}{ Income in $2002^{\mathrm{a}}$} \\
\hline$<\$ 50,000$ & 3,402 & 22.2 \\
\hline$\$ 50,000$ to $<\$ 75,000$ & 3,808 & 24.9 \\
\hline$\$ 75,000$ to $<\$ 150,000$ & 6,954 & 45.5 \\
\hline$\geq \$ 150,000$ & 1,127 & 7.4 \\
\hline \multicolumn{3}{|l|}{ Pre-911 MH diagnosis ${ }^{\mathrm{a}}$} \\
\hline Yes & 1,214 & 7.7 \\
\hline No & 14,515 & 92.3 \\
\hline \multicolumn{3}{|c|}{ Doctor/medication for $\mathrm{MH}$ problem ${ }^{\mathrm{a}}$} \\
\hline Yes & 5,263 & 32.6 \\
\hline No & 10,900 & 67.4 \\
\hline \multicolumn{3}{|l|}{$\geq 1$ traumatic life event pre-9/11 ${ }^{\mathrm{a}, \mathrm{b}}$} \\
\hline Yes & 7,458 & 47.2 \\
\hline No & 8,334 & 52.8 \\
\hline \multicolumn{3}{|l|}{ Dust cloud exposure $^{a}$} \\
\hline None & 10,040 & 66.0 \\
\hline Some & 1,440 & 9.5 \\
\hline Intense & 3,726 & 24.5 \\
\hline \multicolumn{3}{|l|}{ Worked on dust/debris pile ${ }^{\mathrm{a}}$} \\
\hline Yes & 7,484 & 45.5 \\
\hline No & 8,964 & 54.5 \\
\hline \multicolumn{3}{|l|}{ Present in building that collapsed ${ }^{\mathrm{a}}$} \\
\hline Yes & 426 & 2.6 \\
\hline No & 15,948 & 97.4 \\
\hline
\end{tabular}

Note. Numbers in table may not equal 16,488 due to missing data; $\mathrm{MH}=$ mental health.

${ }^{\text {a Total }} n$ : educational attainment $(n=16,409)$, income $(n=15,291)$, pre-9/11 mental health diagnosis $(n=15,729)$, doctor/medication for mental health problem $(n=16,163)$, traumatic life event pre-9/11 $(n=15,792)$, dust cloud exposure $(n=15,206)$, worked on debris pile $(n=16,488)$, present in building that collapsed $(n=15,948) .{ }^{\mathrm{b}} \mathrm{Natural} /$ human-made disaster; serious accident, attack with weapon, attack without weapon with intent to kill/seriously injure; unwanted sexual contact by force or threat of force; other situation in which feared or were seriously injured/killed; witness others in situation in which feared or were seriously injured/killed. 
.96 , overall diagnostic efficiency as high as .94, and sensitivity and specificity as high as .97 and .87 , respectively (Norris \& Hamblen, 2004). Cronbach's $\alpha$ coefficients for the PCL in the study sample were $.94, .95$, and .95 at Waves 1,2 , and 3, respectively.

Covariates included age, race/ethnicity, household income in 2002 , education attained by Wave 1 , self-report of a pre-9/11 diagnosis of PTSD, another anxiety disorder, or depression, and having experienced $\geq 1$ of several specific traumatic events prior to 9/11. Measures of 9/11-related exposures included timing and duration of work; work on the pile; a trichotomous measure of exposure to the ensuing dust cloud based on experiences while in the cloud (none, some, intense); having lost a job due to $9 / 11$; having feared for life or safety; lost a friend, co-worker, or acquaintance; lost a family member or best friend; sustained an injury; or witnessed $\geq$ three of the following traumatic events on 9/11: an airplane hitting the WTC, buildings collapsing, people running from a cloud of smoke, people falling or jumping from the WTC towers, people injured or killed.

Time-dependent covariates, assessed on at least two occasions, included mode of survey completion (telephone, web, mail), marital status, employment status, and a trichotomous measure of social support (low, medium, high).

\section{Data Analysis}

Trajectory group analysis was used to identify groups of individuals sharing similar trajectories of PCL scores over the study period. This approach has been applied to a range of psychiatric outcomes, including courses of PTSD in children (Le Brocque, Hendrikz, \& Kenardy, 2010), prognosis of depression (Colman et al., 2011), and treatment response in schizophrenia (Levine \& Leucht, 2014). Using the PROC TRAJ macro (Jones, Nagin, $\&$ Roeder, 2001) for SAS software (version 9.2) and specifying a censored normal distribution, an initial model was run with a single trajectory group, after which successive models included one additional group at a time. Most (92\%) subjects had PCL scores at all three waves; subjects missing a single score were included in analyses, as PROC TRAJ assumes values of the dependent variable are missing at random, and uses all available values for each subject. Subjects missing relevant independent variables were excluded from analyses; among variables with missing values, degree of dust cloud exposure had the most $(n=1,282)$, whereas witnessing traumatic events on $9 / 11 \mathrm{had}$ the least $(n=25)$; analytic samples ranged in size from 13,636 to the full sample of 16,488 , depending on covariates included. Bayesian information criterion (BIC) values were compared between each model and the one preceding it; the relative value of including additional groups was assessed on the basis of recommended guidelines for BIC differences, group sizes, and average posterior probabilities (Andruff, Carraro, Thompson, Gaudreau, \& Louvet, 2009; Nagin, 2005). A final model was selected that was parsimonious yet plausible and interpretable given a priori knowledge of the distribution of scores in the sample. Significant (at $p<.05$ ) linear and/or quadratic coefficients from equations describing each trajectory were used to define trajectory shape in models for subsequent analyses. Subjects were assigned to the trajectory group for which their probability of membership was highest. The final trajectory pattern was tested on each of the eight occupational subsamples.

Time-stable covariates that significantly $($ at $p<.05)$ differentiated trajectory groups with higher intercepts from that with the lowest intercept, and time-dependent covariates with significant effects on PCL scores within groups in bivariate analyses were included in multinomial logit and multivariate regression models. Because bivariate associations were similar across occupational subsamples, a single multivariate model was run that included all rescue and recovery workers, except where specific hypotheses were being tested. Results of multivariate analyses were interpreted as likelihoods of membership in each higher intercept group relative to the lowest intercept group for time-stable covariates, and as magnitude of change in PCL scores within trajectory groups for timedependent covariates. Additional analyses compared groups in which PCL scores were comparable at initial assessment, but diverged over time to identify predictors associated with the more favorable (remaining or becoming less symptomatic) trajectory.

Approval for collection and analysis of data used for this study was granted by the institutional review boards of the

Table 2

Trajectories of PTSD: Mean PCL Score at Waves 1, 2, and 3, With Intercept, Linear, and Quadratic Parameter Estimates

\begin{tabular}{|c|c|c|c|c|c|c|c|}
\hline \multirow[b]{2}{*}{ Group } & \multirow[b]{2}{*}{$n$} & \multirow[b]{2}{*}{$\%$} & \multicolumn{3}{|c|}{ Mean PCL } & \multicolumn{2}{|c|}{ Parameter estimates } \\
\hline & & & Wave 1 & Wave 2 & Wave 3 & Linear & Quadratic \\
\hline Low-stable & 8,785 & 53.3 & 20.3 & 22.4 & 22.1 & $0.39^{* *}$ & $-0.05^{* *}$ \\
\hline Moderate-stable & 4,729 & 28.7 & 30.2 & 34.7 & 33.4 & $2.35^{* *}$ & $-0.26^{* *}$ \\
\hline Moderate-increasing & 1,053 & 6.4 & 34.0 & 50.9 & 57.0 & $7.75^{* *}$ & $-0.62^{* *}$ \\
\hline High-decreasing & 1,269 & 7.7 & 48.9 & 49.4 & 43.2 & $0.73^{*}$ & $-0.20^{* *}$ \\
\hline High-stable & 652 & 4.0 & 60.7 & 67.9 & 65.5 & $3.73^{* *}$ & $-0.41^{* *}$ \\
\hline
\end{tabular}

Note. $\mathrm{PTSD}=$ posttraumatic stress disorder; $\mathrm{PCL}=\mathrm{PTSD}$ Checklist.

${ }^{*} p<.05 .{ }^{* *} p<.01$. 

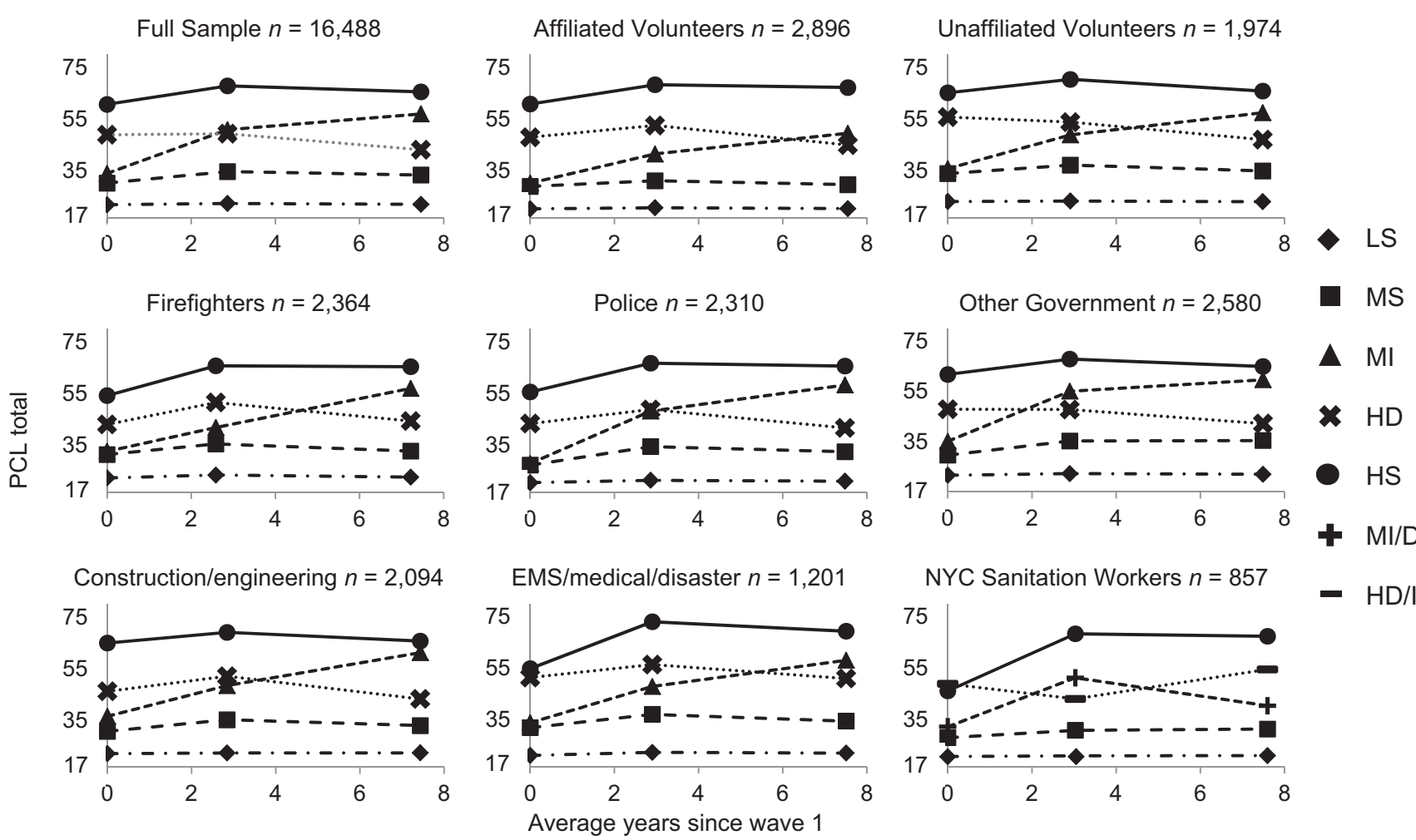

Figure 1. PTSD Checklist Score trajectories by average years since Wave 1 for full sample and occupational subsamples. $\mathrm{LS}=$ low-stable; MS $=$ moderate-stable; $\mathrm{MI}=$ moderate-increasing; $\mathrm{HD}=$ high-decreasing; $\mathrm{HS}=$ high-stable; $\mathrm{MI} / \mathrm{D}=$ moderate increasing/decreasing; $\mathrm{HD} / \mathrm{I}=$ high decreasing/increasing .

Centers for Disease Control and Prevention and the NYC Department of Health \& Mental Hygiene.

\section{Results}

A five-trajectory pattern best described the course of PCL scores over the study period. Not only was the BIC for five trajectories $(-166,134.2)$ lower in absolute value than that for four $(\mathrm{BIC}=-166,774.6)$ or fewer trajectories, but solutions with fewer than five trajectories forced consistently high and consistently low scores onto trajectories with scores that changed substantially over time, thereby accounting for neither the stability nor the variability in the true distribution of scores. Allowing consistently high and consistently low scores to form separate trajectories was therefore given priority over a more balanced solution, in which at least $5 \%$ of the sample would be assigned to each trajectory group. Although BIC values were lower, solutions with six $(\mathrm{BIC}=-165,799.3)$ or more trajectories were less interpretable, and afforded little additional insight into underlying latent classes characterizing the sample.

Table 2 describes the five trajectories by mean PCL values at Waves 1,2, and 3, and linear and quadratic parameters. Just over half of the sample (53.3\%) comprised the low-stable group, in which average PCL scores began (intercept $=20.3$ ), and remained low. Close to one third $(28.7 \%)$ comprised the moderate-stable group, with somewhat higher initial scores (intercept $=30.2)$ that remained relatively stable. The moderateincreasing group was considerably smaller (6.4\%), with scores that also began at a moderate level (intercept $=34.0$ ) and increased over time. The high-decreasing group, which comprised $7.7 \%$ of the sample, had average scores that were initially high (intercept $=48.9$ ) and decreased, while the remaining $4.0 \%$ of the sample comprised the high-stable group, in which scores were consistently high throughout the study period (intercept $=60.7$ ).

Figure 1 depicts the trajectory patterns for the sample and subsamples. As occurred in the full sample, a low-stable group, with intercepts no higher than 23, comprised the largest proportion of each subsample (41.1\%-63.2\%), whereas a high-stable group, with intercepts no lower than 54, included the smallest proportions $(2.3 \%-5.3 \%)$. In all but one subsample (sanitation), remaining groups could be described as moderate-stable, moderate-increasing, and high-decreasing, and included comparable proportions of workers $(22.7 \%-35.9 \%, 4.4 \%-9.0 \%$, and $6.3 \%-12.5 \%)$, respectively.

Adjusted odds ratios (AORs) for 9/11-related exposures significantly associated with membership in higher-intercept trajectory groups, relative to the low-stable group, are shown in Table 3. With the exception of having lost a friend, colleague, or acquaintance, probability of membership in the moderatestable, moderate-increasing, and high-stable groups increased with increasing intercept; probabilities of membership 


\begin{tabular}{|c|c|c|c|c|c|c|c|c|c|c|}
\hline \multirow[b]{2}{*}{ Variable } & \multirow[b]{2}{*}{$n$} & \multirow[b]{2}{*}{$\%$} & \multicolumn{2}{|r|}{$\begin{array}{l}\text { Moderate- } \\
\text { stable }\end{array}$} & \multicolumn{2}{|r|}{$\begin{array}{l}\text { Moderate- } \\
\text { increasing }\end{array}$} & \multicolumn{2}{|r|}{$\begin{array}{c}\text { High- } \\
\text { decreasing }\end{array}$} & \multicolumn{2}{|r|}{ High-stable } \\
\hline & & & $A O R$ & $95 \% \mathrm{CI}$ & $A O R$ & $95 \% \mathrm{CI}$ & $A O R$ & $95 \% \mathrm{CI}$ & $A O R$ & $95 \% \mathrm{CI}$ \\
\hline \multicolumn{11}{|l|}{ First work period } \\
\hline 9/18/2001-6/30/2002 & 4,750 & 29.0 & Ref & & & & & & & \\
\hline 9/11-9/12, 2001 & 8,188 & 49.9 & 1.77 & {$[1.50,2.10]$} & 1.86 & {$[1.48,2.34]$} & 1.32 & {$[1.03,1.70]$} & 1.93 & {$[1.44,2.58]$} \\
\hline 9/13-9/17, 2001 & 3,466 & 21.1 & 1.59 & {$[1.32,1.92]$} & 1.73 & {$[1.34,2.24]$} & 1.56 & {$[1.19,2.05]$} & 2.01 & {$[1.45,2.78]$} \\
\hline \multicolumn{11}{|l|}{ Number of days worked } \\
\hline $1-7$ & 5,523 & 34.7 & Ref & & & & & & & \\
\hline$>7-28$ & 4,962 & 31.2 & 1.07 & {$[0.91,1.25]$} & 1.27 & {$[1.03,1.56]$} & 1.14 & {$[0.90,1.45]$} & 1.50 & {$[1.15,1.95]$} \\
\hline$>28$ & 5,415 & 34.1 & 1.31 & {$[1.11,1.53]$} & 1.73 & {$[1.41,2.12]$} & 1.69 & {$[1.35,2.13]$} & 2.06 & {$[1.60,2.65]$} \\
\hline \multicolumn{11}{|l|}{ Witnessed $\geq 3$ events } \\
\hline No & 12,214 & 74.2 & Ref & & & & & & & \\
\hline Yes & 4,249 & 25.8 & 1.36 & {$[1.14,1.62]$} & 1.78 & {$[1.46,2.17]$} & 1.60 & {$[1.27,2.02]$} & 2.08 & {$[1.64,2.65]$} \\
\hline \multicolumn{11}{|l|}{ Injured on 9/11 } \\
\hline No & 13,836 & 84.2 & Ref & & & & & & & \\
\hline Yes & 2,591 & 15.8 & 1.38 & {$[1.11,1.71]$} & 2.14 & {$[1.71,2.69]$} & 2.01 & {$[1.55,2.61]$} & 2.31 & {$[1.77,3.02]$} \\
\hline \multicolumn{11}{|l|}{ Feared injured/killed } \\
\hline No & 9,734 & 60.2 & Ref & & & & & & & \\
\hline Yes & 6,438 & 39.8 & 2.24 & {$[1.92,2.62]$} & 3.58 & {$[2.95,4.34]$} & 2.70 & {$[2.17,3.35]$} & 5.15 & {$[4.03,6.59]$} \\
\hline \multicolumn{11}{|l|}{ Bereft of } \\
\hline None & 6,592 & 40.0 & Ref & & & & & & & \\
\hline Friend/coworker acquaintance & 7,877 & 47.8 & 1.67 & {$[1.44,1.94]$} & 1.57 & {$[1.29,1.92]$} & 2.01 & {$[1.60,2.52]$} & 1.61 & {$[1.24,2.07]$} \\
\hline Family/best friend & 2,019 & 12.3 & 2.97 & {$[2.30,3.84]$} & 3.59 & {$[2.66,4.84]$} & 4.20 & {$[3.01,5.87]$} & 4.70 & {$[3.31,6.67]$} \\
\hline \multicolumn{11}{|l|}{ Lost job due to $9 / 11$} \\
\hline No & 14,830 & 94.1 & Ref & & & & & & & \\
\hline Yes & 928 & 5.9 & 2.44 & {$[1.56,3.82]$} & 7.77 & {$[5.15,11.72]$} & 5.24 & {$[3.29,8.36]$} & 15.77 & {$[10.28,24.17]$} \\
\hline
\end{tabular}

Note. $\mathrm{PTSD}=$ posttraumatic stress disorder; $A O R=$ adjusted odds ratio; $\mathrm{CI}$, confidence interval; Ref $=$ reference group. All analyses were relative to mild-stable group and adjusted for gender, age, race/ethnicity, educational attainment, pre-9/11 mental health diagnosis, and $\geq 1$ pre-9/11 traumatic event.

in the high-decreasing group were comparable to, or lower than those for the group with the intercept immediately below (the moderate-increasing group). AORs were higher for earlier start dates in all but the high-decreasing group, and increased with days worked and level of bereavement. In all groups, AORs for having lost a family member or best friend were significantly higher than those for having lost a friend, colleague, or acquaintance. Intense exposure to the dust cloud was significantly associated with membership in only the highestintercept group (data not shown).

Time-dependent covariates significantly associated with PCL scores in one or more trajectory groups are shown in Table 4. In comparison to being married or cohabiting, having never been married was associated with significantly higher scores in the low-stable, and significantly lower scores in the high-decreasing group. Having been divorced, widowed, or separated was associated with significantly higher scores in all but the low-stable group, as was having been unemployed. Having reported a low or medium versus a high level of social support was associated with significantly higher PCL scores in all groups. The effect of low social support on PCL scores was not only larger than that of having more social support, but larger than that of any other time-dependent covariate.

There were also some select group comparisons performed. Relative to the moderate-stable group, membership in the moderate-increasing group was more likely for all 9/11-related exposures, and significantly more likely for having sustained an injury or feared for life or safety on, or lost a job because of 9/11. Significantly higher PCL scores were observed in both groups for all time-dependent covariates except having never been married. Having been divorced, widowed, or separated was significantly more likely (data not shown), and associated with greater increases in PCL scores in the moderateincreasing group. Similarly, greater score increases associated with being unemployed were observed in the moderateincreasing group, in which having been or become unemployed during the study period was significantly more likely (data not shown). 
Table 4

Time-Dependent Covariates and PCL Score: $\beta$ Coefficients by PTSD Trajectory Group

\begin{tabular}{|c|c|c|c|c|c|c|c|c|c|c|c|c|}
\hline \multirow[b]{2}{*}{ Variable } & \multirow[b]{2}{*}{$n$} & \multirow[b]{2}{*}{$\%$} & \multicolumn{2}{|c|}{ Low-stable } & \multicolumn{2}{|c|}{ Mod-stable } & \multicolumn{2}{|c|}{ Mod-increasing } & \multicolumn{2}{|c|}{ Hi-decreasing } & \multicolumn{2}{|c|}{ Hi-stable } \\
\hline & & & $B$ & $S E$ & $B$ & $S E$ & $B$ & $S E$ & $B$ & $S E$ & $B$ & $S E$ \\
\hline \multicolumn{13}{|l|}{ Marital status } \\
\hline Married/cohabiting & 12,027 & 73.3 & Ref & & & & & & & & & \\
\hline $\mathrm{D} / \mathrm{W} / \mathrm{S}$ & 1,891 & 11.5 & 0.43 & 0.25 & 1.32 & $0.36^{* * *}$ & 1.60 & $0.64^{*}$ & 1.51 & $0.74^{*}$ & 1.70 & $0.61^{* *}$ \\
\hline Never married & 2,484 & 15.1 & 0.60 & $0.26^{*}$ & 0.26 & 0.34 & 0.93 & 0.70 & 1.72 & $0.74^{*}$ & 1.37 & 0.75 \\
\hline \multicolumn{13}{|l|}{ Employed } \\
\hline Yes & 14,323 & 86.9 & Ref & & & & & & & & & \\
\hline No & 2,114 & 12.8 & 0.10 & 0.20 & 1.83 & $0.27^{* * *}$ & 4.51 & $0.48^{* * *}$ & 5.77 & $0.66^{* * *}$ & 4.90 & $0.51^{\text {*** }}$ \\
\hline \multicolumn{13}{|l|}{ Social support } \\
\hline High & 6,723 & 42.1 & Ref & & & & & & & & & \\
\hline Medium & 5,768 & 36.2 & 1.36 & $0.17^{* * *}$ & 2.16 & $0.22^{* * * *}$ & 2.69 & $0.48^{* * * *}$ & 3.36 & $0.56^{* * *}$ & 2.61 & $0.57^{* * *}$ \\
\hline Low & 3,463 & 21.7 & 3.05 & $0.26^{* * * *}$ & 6.94 & $0.31^{* * * *}$ & 6.92 & $0.54^{* * * *}$ & 9.99 & $0.63^{* * * *}$ & 7.06 & $0.58^{* * *}$ \\
\hline
\end{tabular}

Note. All analyses adjusted for gender, age, race/ethnicity, education, pre-9/11 mental health diagnosis, pre-9/11 traumatic events, and time at the World Trade Center site. PTSD = posttraumatic stress disorder; $S E=$ standard error; PCL $=$ PTSD Checklist; mod = moderate; hi $=$ high; D/W/S $=$ divorced/widowed/separated. ${ }^{*} p<.05 .{ }^{* *} p<.01 .{ }^{* * *} p<.001$.

Membership in the high-stable group was as, or more likely than in the high-decreasing group for all 9/11-related exposures except having lost a friend, colleague, or acquaintance, for which it was lower. Differences between the two groups were statistically significant for having feared for life or safety on, or lost a job because of 9/11. Significantly higher PCL scores were associated with having been divorced, separated, or widowed in both groups; having never been married was associated with significantly lower scores in the high-decreasing group. Having been unemployed was significantly associated with higher scores in both groups, although the effect was higher in the high-decreasing group, in which likelihood of having been, or become unemployed over the study period was significantly lower (data not shown). Having reported a low or medium level of social support was also significantly associated with higher scores in both groups, with the greater impact being in the high-decreasing group.

\section{Discussion}

In a population exposed to the 9/11 disaster, this study categorized courses of PTSD into five trajectory groups, and demonstrated that both time-stable and time-dependent factors are associated with divergent courses of PTSD. Trajectory group assignment was associated with time-stable covariates, including demographic indicators, exposures incurred on 9/11, and psychosocial factors, in ways consistent with previous longitudinal studies of rescue and recovery workers (Berninger et al., 2010; Cukor et al., 2011; Soo et al., 2011). Scores among workers with higher risk profiles were more likely to follow less favorable trajectories, suggesting a dose-response relationship between exposure level and trajectory group risk. This study also demonstrated effects on PTSD trajectories of changing psychosocial conditions over the study period. Again, covariate profiles indicating higher risk were associated with less favorable courses within trajectory groups.

Between-group comparisons revealed significant associations between membership in each of the higher of two risk groups (the moderate-increasing, when compared to the moderate-stable, and the high-stable, when compared to the high-decreasing) and both perceived threat to life on 9/11, and having lost a job because of 9/11. Although not necessarily a realistic assessment of risk, perceived risk is the basis of emotional reactions, and is an important component in the etiology of PTSD (Michaels et al., 1999). It is a correlate of PTSD in other trauma-exposed populations (Holbrook, Hoyt, Stein, $\&$ Sieber, 2001; Michaels et al., 1999), and in rescue and recovery workers responding to events at the WTC in particular (Fullerton, Ursano, Reeves, Shigemura, \& Grieger, 2006). Associations between perceived threat and unresolved symptoms may become stronger over time (Keane, Marshall, \& Taft, 2006); long-term mental health effects of persistent altered perceptions of safety (Grieger, Fullerton, \& Ursano, 2003), are likely associated with the impairments to functioning in social interactions, usual behaviors and reactions to adverse life events (Adams \& Boscarino, 2006; Ursano, Fullerton, Vance, \& Kao, 1999) affecting PTSD status either directly, or as mediators and/or moderators of associations with other correlates. Job loss as a result of 9/11, also associated with higher risk group membership, was likely to have occurred prior to the study period, suggesting that exacerbation of adverse life events may have differentially predisposed those with event-related job loss to poorer outcomes. Compared with the moderate-stable group, the moderate-increasing group was significantly more likely to 
have been or become unemployed, and/or divorced, widowed, or separated over the study period; for both covariates, the associated impact on PCL scores was up to twice as high in the moderate-increasing group. Both employment and marital status are inherently linked to social, economic, and other resources likely associated with PTSD; changes in either imply changes in access to retirement pensions and/or insurance coverage (although medical treatment through federally funded 9/11 programs became available to some sample members, regardless of need, at different times during the study period), as well as changes in job satisfaction and workplace and/or family social support, which may play a larger role in maintenance than in onset of PTSD (Beaton, Murphy, Pike, \& Corneil, 1997; Robinaugh et al., 2011). It is noteworthy that although levels of social support were initially comparable, the group in which symptomatology improved (high-decreasing) was significantly more likely than the group in which symptomatology worsened (moderate-increasing) to have reported a positive change in social support.

Similar trajectory patterns in all but the smallest subset suggested that development of PTSD may be conceptualized similarly across rescue and recovery workers of various occupations, despite varying prevalence. Perrin et al. (2007) suggested protective effects of prior experience and training as partial explanation of variability in PTSD rates in the same cohort at Wave 1. Beaton, Murphy, Johnson, Pike, and Corneil (1999) found inconsistent associations between PTSD and both amount and type of experience among firefighters. Regardless, neither training and experience nor other possible mechanisms that may have underlaid variability in rates of PTSD appeared to differentially impact trajectories, providing further evidence that factors known to predict postdisaster PTSD onset are not necessarily those that determine its course over time (Galea et al., 2005).

Several findings from this study were consistent with those reported by Pietrzak et al. (2014), who applied similar methodology to samples of police and nontraditional workers. Comparable associations were found between several time-stable covariates and membership in higher risk trajectory groups, although this study did not find compelling evidence in favor of separate four and six trajectory solutions for police and nontraditional responders, respectively, even when restricting analyses to these subsamples. Several methodological differences may explain the disparate findings.

Like much research of its kind, this study relied on a selfreport instrument, the validity of which is unknown in the particular population and circumstances of this study. Other limitations to this study included missing data (up to 7.7\%), as well as loss to follow-up and the possibility of differential rates of participation based on constructs hypothesized to be explanatory. Additional analyses revealed no substantial impact of potentially nonrandom patterns of missing data occurring for either reason. Self-report of exposures, mental health diagnosis, and other covariates may have been subject to recall and other response biases. Timing of traumatic life events could be determined relative to $9 / 11$, but not to each wave of data collection. Measures of other mental health disorders that may have been relevant to the findings were available only at Wave 3 , and detailed measures of type and course of treatment were unavailable. Small numbers in the trajectory groups with the highest intercepts may have precluded validity of tests of statistical significance. The voluntary nature of the Registry and associated selection biases therein may have limited the generalizability of these findings. Assumptions made by the analytic model regarding homogenous trajectories with no individual variance may have misrepresented the number of latent classes, and three assessments may have been insufficient to identify some trends in PCL scores over the study period. Strengths of this study included the size and diversity of the sample, as well as repeated measurements of several time-dependent constructs that have been strongly associated with PTSD.

In conclusion, given its continually evolving course, conceptualization of postdisaster PTSD as distinct classes, about which the effects of both time-stable and time-dependent covariates can be estimated, can enhance public health planning and preparedness efforts. Disasters generally occur without warning, precluding baseline measures of individual risk. Nevertheless, increased knowledge about likely courses of PTSD and their determinants may be applied to estimates of ongoing and future needs, and inform budgeting and appropriation of resources over time, enabling reassignment and allocation of resources to address, for example, responders with moderate symptomatology who may warrant intensive follow-up at a later date, or responders whose high degree of symptomatology may be expected to persist. These findings suggest that planning and preparedness for mental health needs following disasters should assume divergent courses of PTSD based on individual and contextual circumstances. They emphasize the need for periodic, comprehensive screening, with simultaneous assessments of psychological, social, economic, and other determinants to aid in identification of individuals at increased likelihood of developing and/or maintaining a level of PTSD symptomatology warranting continued attention and treatment in the future.

\section{References}

Adams, R. E. \& Boscarino, J. A. (2006). Predictors of PTSD and delayed PTSD after disaster: The impact of exposure and psychosocial resources. Journal of Nervous and Mental Disease, 194, 485-493. doi:10.1097/01.nmd.0000228503.95503.e9

American Psychiatric Association. (2000). Diagnostic and statistical manual of mental disorders (4th ed., text rev.). Washington, DC: Author.

Andruff, H., Carraro, N., Thompson, A., Gaudreau, P., \& Louvet, B. (2009). Latent class growth modeling: A tutorial. Tutorials in Quantitative Methods for Psychology, 5, 11-24.

Beaton, R., Murphy, S., Johnson, C., Pike, K., \& Corneil, W. (1999). Coping responses and posttraumatic stress symptomatology in urban fire service personnel. Journal of Traumatic Stress, 12, 293-308. doi:10.1023/A:1024776509667 
Beaton, R. D., Murphy, S. A., Pike, K. C., \& Corneil, W. (1997). Social support and network conflict in firefighters and paramedics. Western Journal of Nursing Research, 19, 297-313. doi:10.1177/019394599701900303

Berninger, A., Webber, M. P., Cohen, H. W., Gustave, J., Lee, R., Niles, J.K., . . Prezant, D. J. (2010). Trends of elevated PTSD risk in firefighters exposed to the World Trade Center disaster: 2001-2005. Public Health Reports, 125, $556-566$.

Bills, C. B., Levy, N. A., Sharma, V., Charney, D. S., Herbert, R., Moline, J., \& Katz, C. L. (2008). Mental health of workers and volunteers responding to events of 9/11: Review of the literature. Mount Sinai Journal of Medicine, 75, 115-127. doi:10.1002/msj.20026

Bowler, R. M., Harrris, M., Li, J., Gocheva, V., Stellman, S. D., Wilson, K., Alper, H., ... Cone, J. (2012). Longitudinal mental health impact among police responders to the 9/11 terrorist attack. American Journal of Industrial Medicine, 55, 297-312. doi: 10.1002/ajim.22000

Chiu, S., Niles, J. K., Webber, M. P., Zeig-Owens, R., Gustave, J., Lee, R., . . Prezant D. J. (2011). Evaluating risk factors and possible mediation effects in posttraumatic depression and posttraumatic stress disorder comorbidity. Public Health Reports, 126, 201-209.

Colman, I., Naicker, K., Zeng, Y., Ataullahjan, A., Senthilselvan, A., \& Patten, S.B. (2011). Predictors of long-term prognosis of depression. CMAJ : Canadian Medical Association Journal, 183, 1969-1976. doi:10.1503/cmaj.110676

Corrigan, M., McWilliams, R., Kelly, K. J., Niles, J., Cammarata, C., Jones, K.,... Prezant, D. J. (2009). A computerized, self-administered questionnaire to evaluate posttraumatic stress among firefighters after the World Trade Center collapse. American Journal of Public Health, 99(Suppl 3), S702-S709. doi:10.2105/AJPH.2008.151605

Cukor, J., Wyka, K., Mello, B., Olden, M., Jayasinghe, N., Roberts, J.,... Difede, J. (2011). The longitudinal course of PTSD among disaster workers deployed to the World Trade Center following the attacks of September 11th. Journal of Traumatic Stress, 24, 506-514. doi:10.1002/jts.20672

Farfel, M., DiGrande, L., Brackbill, R., Prann, A., Cone, J., Friedman, S., ... Thorpe, L. (2008). An overview of 9/11 experiences and respiratory and mental health conditions among World Trade Center Health Registry enrollees. Journal of Urban Health, 85, 880-909. doi:10.1007/s11524-008$9317-4$

Fullerton, C. S., Ursano, R. J., Reeves, J., Shigemura, J., \& Grieger, T. (2006). Perceived safety in disaster workers following 9/11. Journal of Nervous and Mental Disease, 194, 61-63. doi:10.1097/01.nmd.0000195307. 28743.b2

Galea, S., Nandi, A., \& Vlahov, D. (2005). The epidemiology of posttraumatic stress disorder after disasters. Epidemiologic Reviews, 27, 78-91. doi:10.1093/epirev/mxi003

Grieger, T. A., Fullerton, C. S., \& Ursano, R. J. (2003). Posttraumatic stress disorder, alcohol use, and perceived safety after the terrorist attack on the pentagon. Psychiatric Services, 54, 1380-1382. doi:10.1176/appi.ps.54.10. 1380

Gross, R., Neria, Y., Tao, X. G., Massa, J., Ashwell, L., Davis, K., \& Geyh, A. (2006). Posttraumatic stress disorder and other psychological sequelae among World Trade Center clean up and recovery workers. Annals of the New York Academy of Science, 1071, 495-499. doi:10.1196/annals.1364.051

Holbrook, T. L., Hoyt, D. B., Stein, M. B., \& Sieber, W. J. (2001). Perceived threat to life predicts posttraumatic stress disorder after major trauma: Risk factors and functional outcome. Journal of Trauma, 51, 287-292. 292-283.
Jones, B. L., Nagin, D. S., \& Roeder, K. (2001). A SAS procedure based on mixture models for estimating developmental trajectories. Sociological Methods \& Research, 29, 374-393.

Keane, T. M., Marshall, A. D., \& Taft, C. T. (2006). Posttraumatic stress disorder: Etiology, epidemiology, and treatment outcome. Annual Review of Clinical Psychology, 2, 161-197. doi:10.1146/annurev.clinpsy.2.022305. 095305

Le Brocque, R. M., Hendrikz, J., \& Kenardy, J. A. (2010). The course of posttraumatic stress in children: Examination of recovery trajectories following traumatic injury. Journal of Pediatric Psychology, 35, 637-645. doi:10.1093/jpepsy/jsp050

Levin, S. M., Herbert, R., Moline, J. M., Todd, A. C., Stevenson, L., Landsbergis, P., ... Enright, P. (2004). Physical health status of World Trade Center rescue and recovery workers and volunteers-New York City, July 2002-August 2004. Morbidity and Mortality Weekly Reports, 53, 807-812.

Levine, S. Z. \& Leucht, S. (2014). Treatment response heterogeneity in the predominant negative symptoms of schizophrenia: Analysis of amisulpride vs placebo in three clinical trials. Schizophrenia Research, 156, 107-114. doi:10.1016/j.schres.2014.04.005

Michaels, A. J., Michaels, C. E., Zimmerman, M. A., Smith, J. S., Moon, C. H., \& Peterson, C. (1999). Posttraumatic stress disorder in injured adults: Etiology by path analysis. Journal of Trauma, 47, 867-873.

Murphy, J., Brackbill, R. M., Thalji, L., Dolan, M., Pulliam, P., \& Walker, D.J. (2007). Measuring and maximizing coverage in the World Trade Center Health Registry. Statistics in Medicine, 26, 1688-1701. doi:10.1002/sim.2806

Nagin, D. S. (2005). Group-based modeling of development. Cambridge, MA: Harvard University Press.

Norris, F. H. \& Hamblen, J. L. (2004). Standardized self-report measures of civilian trauma and PTSD. In J. P. Wilson, T. M. Keane, \& T. Martin (Eds.), Assessing psychological trauma and PTSD (pp. 63-102). New York, NY: Guilford Press.

Perrin, M. A., DiGrande, L., Wheeler, K., Thorpe, L., Farfel, M., \& Brackbill, R. (2007). Differences in PTSD prevalence and associated risk factors among World Trade Center disaster rescue and recovery workers. American Journal of Psychiatry, 164, 1385-1394. doi:10.1176/appi.ajp.2007.06101645

Pietrzak, R. H., Feder, A., Singh, R., Schechter, C. B., Bromet, E. J., Katz, C. L., ... Southwick, S. M. (2014). Trajectories of PTSD risk and resilience in World Trade Center responders: An 8-year prospective cohort study. Psychological Medicine, 44, 205-219. doi:10.1017/S0033291713000597

Robinaugh, D. J., Marques, L., Traeger, L. N., Marks, E. H., Sung, S. C., Gayle Beck, J., ...Simon, N. M. (2011). Understanding the relationship of perceived social support to post-trauma cognitions and posttraumatic stress disorder. Journal of Anxiety Disordorders, 25, 1072-1078. doi:10.1016/j.janxdis.2011.07.004

Soo, J., Webber, M. P., Gustave, J., Lee, R., Hall, C. B., Cohen, H. W., .. . Prezant, D. J. (2011). Trends in probable PTSD in firefighters exposed to the World Trade Center disaster, 2001-2010. Disaster Medicine and Public Health Preparedness, 5(Suppl 2), S197-S203. doi:10.1001/dmp.2011.48

Ursano, R. J., Fullerton, C. S., Vance, K., \& Kao, T. C. (1999). Posttraumatic stress disorder and identification in disaster workers. American Journal of Psychiatry, 156, 353-359.

Weathers, F. W., Huska, J. A., \& Keane, T. M. (1991). PCL-C for DSM-IV. Boston, MA: National Center for PTSD - Behavioral Science Division. 